\title{
Factores subjetivos asociados a la no adherencia a la CPAP en pacientes con síndrome de apnea hipopnea de sueño
}

\author{
Subjective factors associated with CPAP non- \\ adherence in patients with sleep apnea hypopnea \\ syndrome
}

\author{
María Angélica Bazurto, Karen Herrera, Leslie Vargas, \\ Élida Dueñas, Mauricio González-García • Bogotá, D.C. (Colombia)
}

\section{Resumen}

Introducción: los beneficios de la CPAP dependen del número de horas que el paciente la use. El objetivo del estudio fue establecer, en pacientes con apnea del sueño (SAHS), si hay adecuada adherencia a la CPAP y determinar qué factores referidos por el paciente se relacionan a la no adherencia.

Métodos: estudio observacional analítico transversal en pacientes tratados con CPAP. Se definió buena adherencia como uso mínimo de cuatro horas al menos el 70\% de los días (registro del dispositivo). Análisis de regresión logística para evaluar los factores relacionados a la no adherencia: presión, resequedad de la vía aérea, problemas con la máscara y falta de educación en el uso de CPAP.

Resultados: de 160 pacientes, 88 (55\%) tuvieron mala adherencia. La edad, peso, talla, cuello e índice de apneas fueron similares en los grupos con buena y mala adherencia. El Epworth y la presión de CPAP fueron significativamente mayores en el grupo de mala adherencia $(\mathrm{p}<0.05)$. El promedio de horas de uso de CPAP en el grupo con buena adherencia fue de $5.1 \pm 1.7$ horas y en el grupo con mala adherencia fue de $1.9 \pm 1.5$ horas. Los pacientes sobreestimaron el uso de la CPAP en 2.0 horas: reportadas por el paciente 5.3 horas frente a 3.3 horas según la tarjeta del equipo $(\mathrm{p}<0.001)$. El único factor referido por el paciente que se relacionó con la mala adherencia fue la presión de CPAP (OR ajustado: $3,34[1,34$ a 8,30$])$.

Conclusiones: la adherencia al tratamiento con CPAP en pacientes con SAHS es subóptima (mala adherencia en $55 \%$ de los pacientes). La principal causa relacionada con la no adherencia según los pacientes fue la intolerancia a la presión del dispositivo. Los pacientes sobreestiman las horas reales de uso del CPAP. (Acta Med Colomb 2013; 38: 71-75).

Palabras clave: apnea obstructiva de sueño, presión positiva continua en la vía aérea, CPAP, adherencia, tratamiento.

\section{Abstract}

Introduction: the benefits of CPAP depend on the number of hours being used by the patient. The aim of the study was to establish, in patients with sleep apnea (SAHS), if there is adequate adherence to CPAP and to determine what factors reported by the patient are related to non-adherence.

Methods: an analytical observational cross-sectional study in patients treated with CPAP. Good adherence was defined as a minimum of 4 hours use at least $70 \%$ of days (device score). Logistic regression analysis to assess factors related to non-adherence: pressure, airway dryness, mask problems and lack of education in the use of CPAP.

Results: of 160 patients, 88 (55\%) had poor adherence. Age, weight, height, neck and apnea index were similar in the groups with good and poor adherence. The Epworth and CPAP pressure were significantly higher in the group of poor adherence $(\mathrm{p}<.05)$. The average hours of use of CPAP in the group with good adherence was $5.1 \pm 1.7$ hours and the poor adherence group was $1.9 \pm 1.5$ hours. The patients overestimated the use of CPAP in 2.0 hours: 5.3 hours reported by the patient versus 3.3
Dra. María Angélica Bazurto Z.: Internista Neumóloga, Servicio de Trastornos Respiratorios del Sueño; Karen Herrera N.: Fisioterapeuta Servicio de Trastornos Respiratorios del Sueño; Dra. Leslie Vargas: Internista Neumóloga, Servicio de Trastornos Respiratorios del Sueño; Dra. Élida Dueñas M.: Pediatra Neumóloga, Servicio de Trastornos Respiratorios del Sueño; Dr. Mauricio González-García: Internista, Neumólogo, Epidemiólogo, Departamento de Investigación. Fundación Neumológica Colombiana. Bogotá, D.C. (Colombia).

Correspondencia. Dra. María Angélica Bazurto Z.

E-mail: mbazurto@neumologica.org Recibido: 26/III/2012 Aceptado: 7/V/2013 
hours according to the device card $(\mathrm{p}<0.001)$. The only factor reported by the patient that was associated with poor adherence was CPAP pressure (adjusted OR: 3.34 [1.34 to 8.30]).

Conclusions: adherence to CPAP in patients with SAHS is suboptimal (poor adherence in 55\% of patients). The main cause related to non-adherence according to patients was intolerance to the pressure device. Patients overestimate the real hours of CPAP use. (Acta Med Colomb 2013; 38: 71-75)

Keywords: obstructive sleep apnea, continuous positive pressure in the airway, CPAP, adherence, treatment.

\section{Introducción}

El síndrome de apnea hipopnea de sueño (SAHS) es una condición frecuente en adultos (4\% en hombres y $2 \%$ en mujeres) (1) y se asocia con morbilidad cardiovascular y mortalidad entre individuos no tratados (2). La presión positiva continua en la vía aérea (CPAP) ha sido el tratamiento más efectivo en pacientes con SAHS moderado o severo, aunque también se han visto algunos beneficios en SAHS leve (3). El tratamiento con CPAP mejora la somnolencia diurna $(4,5)$, disminuye los accidentes de tránsito (6), contribuye al control de la hipertensión arterial $(7,8)$ y disminuye la ocurrencia de eventos cardiovasculares fatales y no fatales (9).

La mayoría de los estudios han mostrado que algunos beneficios de la CPAP, como la mejoría de la somnolencia, del rendimiento diario y la memoria, dependen de las horas de uso (10). Sin embargo, la adherencia a este tratamiento no es óptima. Solamente $46-80 \%$ de los pacientes se adhieren al tratamiento y $8-15 \%$ rehúsan a usar el dispositivo después de la noche de titulación (11-13).

El objetivo del estudio fue establecer, en un grupo de pacientes con SAHS, la adherencia a la CPAP y los factores referidos por los pacientes y relacionados con la no adherencia.

\section{Material y métodos}

Estudio observacional analítico de corte transversal en pacientes con SAHS en tratamiento con dispositivos de CPAP, residentes en Bogotá ( $2640 \mathrm{~m}$ sobre el nivel del mar), que fueron remitidos a la consulta de trastornos respiratorios del sueño de la Fundación Neumológica Colombiana. Se incluyeron pacientes que asistieron con el dispositivo, la máscara y los estudios polisomnográficos basal y de titulación, entre el 15 de abril y el 15 de junio de 2010. Se excluyeron pacientes con datos incompletos en el formulario de recolección de datos realizado en la consulta o en los estudios polisomnográficos, aquellos con dispositivos de presión positiva que no permitían establecer el tiempo de uso y pacientes con menos de un mes de uso de CPAP. Se realizó una historia clínica completa dirigida a síntomas de apnea del sueño, hábitos de sueño, tiempo de uso de CPAP (noches por semana, horas por noche), efectos secundarios y las principales molestias que limitaban el uso de la CPAP.

En cuanto al uso de CPAP se preguntó específicamente: ¿Cuántas noches por semana usa la CPAP?, ¿cuántas horas por noche usa la CPAP?, ¿cuál es la principal molestia que hace que usted no use la CPAP continuamente? Estas molestias fueron divididas en cinco categorías: 1. Máscara: molestias derivadas de la máscara como sitios de presión, irritación de la piel, fugas orales, otras fugas. 2. Vía aérea: definida como obstrucción nasal, resequedad en boca y/o nariz. 3. Presión de CPAP: intolerancia a la presión generada por el aparato, dificultad en la exhalación, dificultad para respirar por la presión. 4. Educación: falta de información con respecto al uso del equipo o la máscara, no clara conciencia de las consecuencias de la enfermedad y de los beneficios del uso de la CPAP. 5. Otros: claustrofobia, insomnio y cefalea.

En los aparatos que tenían la opción, se descargó el informe obteniendo los siguientes datos: días totales evaluados, promedio de horas de uso y porcentaje de días que usó el dispositivo por más de 4 horas. De los otros dispositivos se tomó el total de las horas de uso y para obtener el porcentaje se dividieron por el número total de días de uso (14). Se definió buena adherencia como el uso de CPAP por un mínimo de cuatro horas al menos el 70\% de los días (12). El estudio fue aprobado por el Comité de Ética de la institución.

\section{Análisis estadístico}

Se realizó una descripción de la muestra utilizando promedios y desviación estándar para las variables cuantitativas y proporciones para las variables cualitativas. Para evaluar diferencias entre los pacientes con y sin buena adherencia al dispositivo de presión, se utilizó la prueba $t$ de Student para las variables continuas y el estadístico chi cuadrado para las variables cualitativas. Se utilizó un análisis de regresión logística para evaluar la asociación entre la adherencia a la CPAP y las variables referidas por el paciente. Se formularon hipótesis a dos colas y se tomó un valor de p menor a 0.05 como significativo. Se utilizó el software estadístico SPSS versión 15 .

\section{Resultados}

Se incluyeron 160 pacientes, de los cuales 51.9\% fueron hombres. Según la definición usada, 88 pacientes (55\%) tuvieron mala adherencia a la CPAP. No hubo diferencias en la adherencia entre las dos formas de medición de las horas de uso de la CPAP dependiendo del tipo de dispositivo: $55.1 \%$ vs $54.9 \%(\mathrm{p}=0.987)$. Al compararlo con el grupo con buena adherencia, el grupo con mala adherencia tuvo una escala de somnolencia de Epworth medida en el momento de la 
Tabla 1. Características de los pacientes.

\begin{tabular}{|c|c|c|c|c|}
\hline & $\begin{array}{l}\text { Grupo total } \\
n=160\end{array}$ & $\begin{array}{l}\text { Mala adherencia } \\
\qquad \mathbf{n}=\mathbf{8 8}\end{array}$ & $\begin{array}{l}\text { Buena adherencia } \\
\qquad \mathbf{n}=72\end{array}$ & $\mathbf{p}^{*}$ \\
\hline Edad, años & $58.8 \pm 10.7$ & $58.7 \pm 10.4$ & $58.9 \pm 11.1$ & 0.922 \\
\hline Peso, $\mathrm{Kg}$ & $81.8 \pm 18.1$ & $83.0 \pm 21.6$ & $80.3 \pm 12.5$ & 0.325 \\
\hline Talla, m & $1.61 \pm 0.09$ & $1.62 \pm 0.10$ & $1.60 \pm 0.90$ & 0.484 \\
\hline $\mathrm{IMC}, \mathrm{Kg} / \mathrm{m}^{2}$ & $31.8 \pm 5.8$ & $32.4 \pm 6.5$ & $31.1 \pm 4.8$ & 0.136 \\
\hline Cuello, cm & $41.6 \pm 4.3$ & $41.7 \pm 4.5$ & $41.6 \pm 4.0$ & 0.973 \\
\hline IAH & $51.9 \pm 28.6$ & $52.8 \pm 30.3$ & $50.8 \pm 26.6$ & 0.651 \\
\hline Presión C-PAP, cm agua & $10.5 \pm 3.4$ & $11.1 \pm 3.5$ & $9.9 \pm 3.1$ & 0.020 \\
\hline $\mathrm{SpO}_{2}$ PSG basal, $\%$ & $78.3 \pm 9.3$ & $78.5 \pm 9.0$ & $78.0 \pm 9.8$ & 0.727 \\
\hline $\mathrm{SpO}_{2}$ PSG con CPAP, $\%$ & $83.5 \pm 20.7$ & $84.3 \pm 19.7$ & $82.4 \pm 22.1$ & 0.589 \\
\hline Escala de Epworth & $8.3 \pm 5.2$ & $9.5 \pm 5.4$ & $6.8 \pm 4.5$ & 0.001 \\
\hline Meses de uso del CPAP & $16.9 \pm 14.1$ & $14.6 \pm 12.3$ & $19.8 \pm 15.6$ & 0.022 \\
\hline Horas de uso del CPAP & $3.3 \pm 2.3$ & $1.9 \pm 1.5$ & $5.1 \pm 1.7$ & $<0.001$ \\
\hline
\end{tabular}

evaluación y una presión de CPAP significativamente más altas y un tiempo de uso significativamente menor (Tabla 1). La mayoría de los pacientes usaban máscara nasal (63.8\%), $53.8 \%$ tenían humidificador y $40.6 \%$ usaban oxígeno con la CPAP, sin diferencias entre los grupos.

El tiempo de uso de la CPAP desde su prescripción fue variable. El $18.8 \%$ de los pacientes llevaba menos de seis meses con la CPAP, $39.4 \%$ entre seis y 12 meses, y $41.8 \%$ más de 12 meses. La mala adherencia fue mayor en los pacientes que venían utilizando el tratamiento con CPAP por menos de 12 meses en comparación con quienes lo estaban utilizando por más de 12 meses (62.4 Vs. 44.8\%, p= 0.020).

El promedio de horas de uso de la CPAP, según el registro de la tarjeta, fue de $5.1 \pm 1.7$ horas en el grupo con buena adherencia y de $1.9 \pm 1.5$ en el grupo con mala adherencia. Al hacer la comparación entre las horas de uso de la CPAP reportadas por el paciente con las horas reales de uso del equipo según la tarjeta, los pacientes sobreestimaron el uso de la CPAP en 2.0 horas: $5.3 \pm 2.3$ frente a $3.3 \pm 2.3(\mathrm{p}<0.05)$ (Figura 1).

La queja más frecuente de los pacientes fue la molestia derivada de la máscara (66.9\%), seguida de los síntomas de vía aérea superior (27.6\%). En el análisis multivariado (Tabla 2), el único factor referido por el paciente, que se relacionó con la mala adherencia fue la variable presión de CPAP con un OR ajustado de 2.69 (IC 95\% de 1.18 a 6.1).

\section{Discusión}

La mala adherencia al tratamiento con CPAP en este estudio fue alta (55\%) y el único factor referido por los pacientes que se relacionó significativamente con esta mala adherencia fue la molestia producida por la presión del dispositivo de CPAP. Adicionalmente encontramos que los

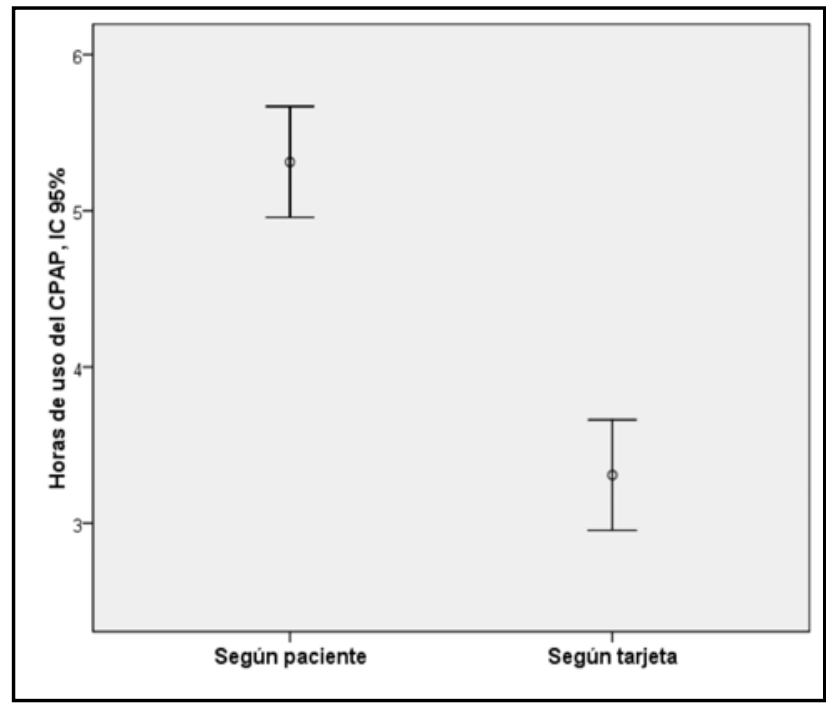

Figura 1. Horas de uso de la CPAP referidas por el paciente y según la lectura de la tarjeta $5.3 \pm 2.3$ Vs. $3.3 \pm 2.3(p>0.05)$.

Tabla 2. Factores referidos por los pacientes relacionados con la adherencia a la CPAP.

\begin{tabular}{|l|c|c|}
\hline Variable & OR crudo & OR ajustado \\
\hline Máscara & $1.60(0.83$ a 3.11$)$ & $1.62(0.81$ a 3.25$)$ \\
Vía aérea & $1.26(0.62$ a 2.54$)$ & $1.47(0.71$ a 3.05$)$ \\
Presión de CPAP & $2.60(1.16$ a 5.84$)$ & $2.69(1.18$ a 6.15$)$ \\
Educación & $1.65(0.15$ a 18.59$)$ & $3.25(0.27$ a 38.99$)$ \\
Otros & $1.39(0.32$ a 6.00$)$ & $1.85(0.41$ a 8.39$)$ \\
\hline Definiciones en el texto
\end{tabular}


pacientes sobreestiman el uso de la CPAP en comparación con el tiempo real registrado por los dispositivos.

A pesar de que hay beneficios claros con el uso de la CPAP, el hecho que sea un tratamiento de uso continuo, que no sea curativo y que genere molestias hace que la adherencia a él no sea buena. El tratamiento con CPAP no es la excepción entre otros tratamientos crónicos; de acuerdo con la Organización Mundial de la Salud, 50\% de los pacientes con enfermedades crónicas no cumplen con el tratamiento, ya sea farmacológico o basado en cambios en el estilo de vida (15) y en pacientes que reciben oxígeno domiciliario a largo plazo, por ejemplo, se ha encontrado que sólo $45 \%$ cumplen la recomendación de uso de éste por 15 horas al día (16).

La definición de adherencia a la CPAP varía en los diferentes estudios. La empleada por nosotros, que es la de uso más frecuente en la literatura, fue establecida arbitrariamente en el trabajo de Kribbs et al (12) como un uso mayor de cuatro horas al menos el $70 \%$ de las noches monitoreadas. Aunque las horas de uso por noche es el parámetro más usado en los estudios de adherencia (17), es importante tener en cuenta la cantidad de noches de uso, ya que como ha sido descrito por Weaver et al (18), el no usarlo algunas noches puede hacer que persistan algunos síntomas de SAHS como somnolencia diurna.

En este estudio medimos las horas de uso de la CPAP de dos formas aceptadas en la literatura. En algunos dispositivos el software establece el tiempo, el número de días y el porcentaje de uso de la CPAP y en otros, se registran las horas de uso, que se dividen por el tiempo de prescripción, método descrito por Rauscher et al (14). Pepin et al, demostraron que la adherencia objetiva (medida por el equipo) y la adherencia efectiva (definida como el tiempo que la máscara de CPAP es efectivamente aplicada), es muy similar (19), por lo que los dos métodos son aceptados para medir la adherencia (20). En este estudio, como ya se mencionó, la mala adherencia fue de 55\% sin diferencias entre las dos formas de medición de las horas de uso de la CPAP, sugiriendo que son comparables. Además, el tener equipos diferentes refleja la práctica clínica diaria en la cual debemos evaluar la adherencia de todos los pacientes.

$\mathrm{Al}$ igual que en otros estudios, factores como la edad, el sexo, el IMC y el IAH no se relacionaron con la adherencia (19). Los factores determinantes de uso óptimo de la CPAP han sido contradictorios en la literatura. El grado de somnolencia es uno de los factores que con más frecuencia se ha encontrado relacionada con buena adherencia a la CPAP $(21,22)$. La mayor somnolencia encontrada en este estudio en el grupo de mala adherencia, es debida a que la escala de Epworth reportada fue la realizada en el momento de la evaluación y no al iniciar el tratamiento, reflejando probablemente una mayor somnolencia del grupo que no cumple el tratamiento

Los efectos secundarios más frecuentes como molestias en la vía aérea o los asociados con la máscara no se relacionaron en este grupo de pacientes con la adherencia, lo que ya ha sido encontrado en otros estudios $(12,23,24)$. Ni el tipo de máscara ni el uso de humidificador se relacionaron con un mayor o menor uso de la CPAP. A pesar de la cantidad de máscaras disponibles en el mercado, los pocos estudios que han evaluado si el tipo de máscara puede impactar la adherencia a la CPAP en general no han encontrado diferencias significativas (25), a excepción del estudio de Mortimore $e t$ al en el cual se encontró que los pacientes con máscara nasal usan una hora más la CPAP que los que usan máscara oronasal (26). No se ha comprobado que el uso de humidificador mejore el uso. Ryan et al encontraron que el humidificador disminuye los síntomas nasales pero no mejora la adherencia (27) y Mador tampoco demostró mejoría en la adherencia (28).

Con respecto al tiempo de uso, los pacientes con menos de 12 meses de prescripción de la CPAP tuvieron peor adherencia que los que llevaban más tiempo de tratamiento, a diferencia de lo encontrado por La Piana et al, quienes encontraron disminución en el uso de CPAP a los dos años. Estos autores atribuyeron la disminución de la adherencia a la terminación de los programas de educación en uso de la CPAP luego de este tiempo (29), estrategia para mejorar la adherencia no claramente establecida en nuestro medio. La mejor adherencia que encontramos en los pacientes con más de doce meses de uso podría explicarse a que estos sujetos son los realmente adherentes, a diferencia de los que inician el tratamiento y tienen un patrón de uso irregular, que abandonan definitivamente el tratamiento en los primeros meses.

El único factor en este estudio referido por los pacientes con la mala adherencia, fue la molestia derivada de la "presión" de CPAP. Aunque la diferencia en la presión entre los grupos de mala y buena adherencia fue pequeña, fue estadísticamente significativa y clínicamente puede explicarse porque los pacientes con frecuencia encuentran difícil la exhalación en contra de una presión. En los estudios que han evaluado dispositivos diseñados para disminuir la presión en la espiración como el BiPAP y en otros que varían y optimizan la presión como la CPAP automático, no han encontrado que mejoren la adherencia, comparados con la CPAP fija $(30,31)$.

Las horas de uso promedio encontradas en este estudio de $3.3 \pm 2.2$, es uno de los primeros datos de adherencia reportados en Latinoamérica, y es menor de los reportados en países de Europa y en Estados Unidos. Entre los factores que pudieron influenciar esta diferencia, está el modelo de atención específico de Colombia. Sin embargo, no se ha determinado si el estándar de cuidado o la cultura influyen en el cumplimiento al tratamiento. Usamos la definición de adherencia que incluye las horas de uso y el porcentaje del tiempo usado, ya que creemos que los criterios de buena adherencia tienden a ser cada vez más exigentes, debido a la creciente evidencia, que a más tiempo de uso hay más beneficios en síntomas (10) y en desenlaces a largo plazo, como el impacto que tiene el tiempo de uso de la CPAP (más de 5.6 horas) en la disminución de cifras de tensión arterial 
de pacientes con SAHS, hipertensos no somnolientos, encontrada por Barbe et al (7).

$\mathrm{Al}$ igual que otros estudios como el de Rauscher et al (14), encontramos que los pacientes sobreestiman las horas de uso por más de una hora, lo que confirma que la adherencia debe ser medida, de forma objetiva, principalmente en pacientes que no han tenido la respuesta clínica esperada.

Entre las limitaciones del estudio están la inclusión de pacientes con tiempos de uso variables y diferentes "estándares de atención", es decir, los pacientes asistían a diferentes médicos y la forma en la que se inició el tratamiento (incluida la adaptación inicial y la educación) no fue la misma, sin embargo esto refleja la práctica clínica diaria. Adicionalmente, no contábamos con datos previos a la prescripción del tratamiento con CPAP como síntomas o escala de Epworth, los cuales se han relacionado más claramente con los índices de cumplimiento.

En conclusión, estos resultados indican que la adherencia al tratamiento con CPAP en pacientes con SAHS es subóptima, generando importantes costos al sistema de salud. En este grupo de pacientes la principal causa relacionada con la no adherencia fue la intolerancia a la presión de CPAP. En el seguimiento clínico se debe incluir una forma objetiva de medir el cumplimiento ya que los pacientes tienden a sobreestimar el tiempo de uso.

\section{Financiación}

Esta investigación se realizó con recursos de la Fundación Neumológica Colombiana, no recibió aportes de ninguna otra entidad.

\section{Conflictos de interés}

Los autores no recibieron ningún pago por parte de ninguna entidad para efectos de la investigación.

M.A. Bazurto: declaro que no tengo ningún conflicto de interés.

L. Vargas: declaro que no tengo ningún conflicto de interés.

K. Herrera: declaro que no tengo ningún conflicto de interés.

E. Dueñas: declaro haber recibido honorarios por conferencias dictadas a laboratorios Astra Zeneca y Merck. No recibo dinero en forma rutinaria de la industria farmacéutica o de las compañías que promocionan y venden equipos médicos (polisomnógrafos, CPAP, BPAP). No tengo ningún otro conflicto de interés que interfiera con la realización de la presente investigación

M. González: declaro que no tengo ningún conflicto de interés.

\section{Referencias}

1. Young T, Palta M, Dempsey J, Skatrud J, Weber S, Badr S. The occurrence of sleep-disordered breathing among middle-aged adults. N Engl J Med 1993; 328: $1230-5$.

2. Mc Nicholas WT, Bonsignore MR. Sleep apnoea as an independent risk factor for cardiovascular disease: current evidence, basic mechanisms and research priorities Eur Respir J 2007; 29: 156-78.

3. Redline S, Adams N, Strauss ME et al. Improvement of Mild Sleep-disordered Breathing with CPAP Compared with Conservative Therapy. Am J Respir Crit Care Med 1998; 157: 858-65.

4. Engleman HM, Martin SE, Kingshott RN, Mackay TW, Deary IJ, Douglas NJ. Randomised placebo controlled trial of daytime function after continuous positive airway pressure (CPAP) therapy for the sleep apnoea/hypopnoea syndrome. Thorax 1998; 53: 341-5.

5. Patel SR, White DP, Malhotra A, Stanchina ML,Ayas NT. Continuous positive airway pressure therapy for treating sleepiness in a diverse population with obstructive sleep apnea: results of a meta-analysis. Arch Intern Med 2003; 163: 565-71.

6. Teran-Santos J, Jimenez-Gomez A, Cordero-Guevara J. The association between sleep apnoea and the risk of traffic accidents. N Engl J Med 1999; 340 : 847-51.

7. Barbe F, Duran-Cantolla J, Capote F, et al. Long-term effect of continuous positive airway pressure in hypertensive patients with sleep apnea. Am J Respir Crit Care Med 2010; 181: 718-26.

8. Durán-Cantolla J,Aizpuru F, Martínez C, et al. Efficacy of continuous positive pressure (CPAP) on patients with recently diagnosed of system hypertension ( $\mathrm{SH}$ ) and obstructive sleep apnea-hypopnea (OSAH). ATS international Conference Toronto, Ontario, USA. 16-21 May 2008; 177: A805

9. Marin JM, Carrizo S et al. Long-term cardiovascular outcomes in men with obstructive sleep apnoea-hypopnea with or without treatment with continuous positive airway pressure: an observational study. Lancet 2005; 365: 1046-53

10. Weaver TE, Maislin G, Dinges DF, Bloxham T, George CF, Greenberg H, Kader G, Mahowald M, Younger J, Pack AI. Relationship between hours of CPAP use and achieving normal levels of sleepiness and daily functioning. Sleep 2007; 30: 711-9.

11. Stradling JR, Davies RJ. Is more NCPAP better? Sleep 2000; 23: S150-3.

12. Kribbs NB, Pack AI, Kline LR, et al. Objective measurement of patterns of nasal CPAP use by patients with obstructive sleep apnea. Am Rev Respir Dis 1993; 147: 887-95.

13. Rosenthal L, Gerhardstein R, Lumley A, et al. CPAP therapy in patients with mild OSA: implementation and treatment outcome. Sleep Med 2000; 1: 215-20.

14. Rauscher H, Formanek D et al. Self-Reported vs Measured Compliance with Nasal CPAP for Obstructive Sleep Apnea. Chest 1993; 103: 1675-80.

15. Sabate E. Adherence to long-term therapies: evidence for action. Geneva: World Health Organization; 2003. WHO/MNC/03.01.

16. Pépin, J. L., C. E. Barjhoux, C. Deschaux, and C. Brambilla. 1996. Longterm oxygen therapy at home: compliance with medical prescription and effective use of therapy. Chest 1996; 109: 1144-50.

17. Engleman HM, Martin SE, Douglas NJ. Compliance with CPAP therapy in patients with the sleep apnoea/hypopnoea syndrome. Thorax 1994; 49: 263-6.

18. Weaver T, Barone N, Pack A. Night-To-Night Variability in CPAP Use Over the First Three Months of Treatment. Sleep 1997; 20: 278-83.

19. Pepin JL, Kriegere J, Rodenstein D et al. Effective Compliance during the First 3 Months of Continuous Positive Airway Pressure A European Prospective Study of 121 Patients. Am J Respir Crit Care Med 1999; 160: 1124-9.

20. Weaver TE, Sawyer A. Adherence to Continuous Positive Airway Pressure Treatment for Obstructive Sleep Apnea: Implications for Future Interventions Indian J Med Res 2010; 131: 245-258.

21. Waldhorn RE, Herrick TW, Nguyen MC, et al. Long-term compliance with nasal continuous positive airway pressure therapy of obstructive leep apnea. Chest 1990; 97: 33-8

22. Rauscher H, Popp W, Wanke T, et al. Acceptance of CPAP therapy for sleep apnea. Chest 1991; 100: 1019-23

23. Fletcher E, Luckett R. The effect of positive reinforcement on hourly compliance in nasal continuous positive airway pressure users with obstructive sleep apnea. Am Rev Respir Dis 1991; 143: 936-41.

24. Hoffstein V, Viner S, Mateika S, Conway J. Treatment of obstructive sleep apnea with nasal continuous positive airway pressure. Patient compliance, perception of benefits, and side effects. Am Rev Respir Dis 1992; 145: 841-5.

25. Beecroft J,Zanon S et al. Oral Continuous Positive Airway Pressure for Sleep Apnea* Effectiveness, Patient Preference, and Adherence. Chest 2003; 124: 2200-8.

26. Mortimore IL, Whittle AT, Douglas NJ. Comparison of nose and face mask CPAP therapy for sleep apnea. Thorax 1998; 53: 290-2.

27. Ryan S, Doherty L, Nolan G, McNicholas WT. Effects of Heated Humidification and Topical Steroids on Compliance, Nasal Symptoms, and Quality of Life in Patients with Obstructive Sleep Apnea Syndrome Using Nasal Continuous Positive Airway Pressure. J Clin Sleep Med 2009; 5(5): 422-7.

28. Mador MJ, Krauza M, Pervez A, Pierce D, Braun M. Effect of heated humidification on compliance and quality of life in patients with sleep apnea using nasal continuous positive airway pressure. Chest 2005; 128: 2151-8.

29. La Piana GE, Scartabellati A, Chiesa L. Long-term adherence to CPAP treatment in patients with obstructive sleep apnea: importance of educational program. Patient Preference and Adherence 2011; 5: 555-562

30. Gay PC, Herold DL, Olson EJ. A randomized, double-blind clinical trial comparing continuous positive airway pressure with a novel bilevel pressure system for treatment of obstructive sleep apnea syndrome. Sleep 2003; 26: 864-869.

31. Ayas NT, Paytel SR, Malhotra A, Schulzer M, Malhotra M, Jung D, Fleetham J, White DP. Auto-titrating versus standard continuous positive airway pressure for the treatment of obstructive sleep apnea: results of a meta-analysis. Sleep 2004; 27 : 249-253. 